\title{
Psychometric properties of the adolescent stress questionnaire-short form scores and association with self-efficacy
}

\author{
James R Andretta ${ }^{1}$; Michael T McKay ${ }^{2 *}$; Donald G Byrne ${ }^{3}$ \\ ${ }^{1}$ Child Guidance Clinic, Superior Court of the District of Columbia, USA \\ ${ }^{2}$ Department of Psychological Sciences, University of Liverpool, UK \\ ${ }^{3}$ Medical School, The Australian National University, Australia
}

\author{
*Corresponding Author(s): Michael T McKay \\ Department of Psychological Sciences, University \\ of Liverpool, UK
}

Tel: +0044 7875778186

Email: Michael.McKay@liverpool.ac.uk

Received: Mar 01, 2018

Accepted: Jun15, 2018

Published Online: Jun 20, 2018

Journal: Journal of Psychiatry and Behavioral Sciences

Publisher: MedDocs Publishers LLC

Online edition: http://meddocsonline.org/

Copyright: (C) McKay MT (2018). This Article is distributed under the terms of Creative Commons Attribution 4.0 International License

Keywords: Adolescent stress questionnaire; Internal consistency; Scotland; Northern Ireland; Measurement invariance

\section{Abstract}

Adolescence is a period of intense biological, and psychological change. This change can result in stress, which in turn can lead to a range of negative mental and physical health outcomes. It is important that stress in adolescence is measured accurately and reliably. This study examined a new and shortened 24-item version of the Adolescent Stress Questionnaire that accounts for stress in eight domains. Participants were school children in Northern Irish and Scottish high schools who completed a questionnaire 33 months apart. The questionnaire assessed stress, selfefficacy in three domains, and socio-demographic variables. Results of Confirmatory Factor Analyses in part one supported the proposed eight factor structure ASQ scores, as well as measurement invariance across country of residence, gender, socioeconomic status groups, and time. Correlations between scores on the eight factors and scores on three domains of self-efficacy supported construct validity. In short, higher levels of stress in some, but not all domains, was associated with lower social, emotional, and academic self-efficacy. Future studies are recommended to include more exploration into the usefulness of conceptualizing ASQ scores as multivariate. The study demonstrates that shortened ASQ scores are viable and reliable indicators of adolescent stress, and that stress has a negative relationship with the degree to which adolescents feel able to manage major life demands.

\section{Introduction}

Adolescence is a period of intense biological and psychological change that can sometimes result in stress [1], which could have a negative impact on mental well-being [2]. The 56-item Adolescent Stress Questionnaire [1] is an index of stress consisting of items forming 10 different stress components or domains. Psychometric studies examining the properties of ASQ scores have reported mixed results with some supportive [1,3], and others reporting problems with both structural validity and internal consistency [4], test-retest reliability [5] and problematic factor loadings [6]. Although McKay et al. [6] reported adequate fit indicies among Northern Irish adolescents (CFI = .95; RMSEA $=.08)$, these authors also reported concerns about the reliability $(\alpha=.50)$ and validity of 'emerging adult responsibility' scores specifically.

Furthermore, the importance of ASQ domain specificity in

Cite this article: Andretta JR, McKay MT, Byrne DG. Psychometric properties of the adolescent stress questionnaire-short form scores and association with self-efficacy. J Psychiatry Behav Sci. 2018; 2: 1008. 
adolescents has also been demonstrated in multiple studies. That is, relationships among ASQ domains and other variables of consequence were heterogenous: (a) depressive symptoms [7] (b) life satisfaction [8], (c) alcohol use [9], and (d) self-efficacy [10]. While the usefulness of ASQ scores is well established, its cumbersome length remains a practical concern especially when used in research projects involving multiple instruments. The present study was focused on the development of a shortened version of the ASQ.

\section{Stress and self-efficacy}

Research has suggested that self-efficacy beliefs play an important role in stress levels and associated outcomes [11,12], with those higher in self-efficacy reporting lower levels of stress. Roddenberry and Renk (2010) found that those who self-reported higher levels of stress also reported higher levels of external locus of control, lower self-efficacy and higher levels of illness. In a study in Northern Ireland, and controlling for age and gender, McKay et al. [10] performed a series of regression models examining predictors of academic, social, and emotional self-efficacy scores. Higher academic self-efficacy was associated with reduced stress of school attendance and stress of school performance levels, but with increased levels of the stress of peer pressure. By contrast, higher social self-efficacy was associated with lower levels of the stress of peer pressure, and the stress of future uncertainty. Finally, higher emotional self-efficacy was associated with lower stress about peer pressure, but higher stress about both romantic relationships and interactions with school teachers. In summary, there are reasonable grounds to consider self-efficacy as a useful construct with which to examine the convergent validity of ASQ scores.

\section{Participants and procedure}

Data were from two independent samples of school children in the United Kingdom. Schools were randomly chosen to be part of a large randomized controlled trial [26]. The schools (N $=105$ ) in the control and intervention groups of the trial were randomly assigned (1:1) to minor 'studies' examining the development of a number of psychological constructs, among them stress. Data were opportunistically collected on adolescent stress in one fifth $(N=21)$ of these schools. Stress was not a covariate in the analyses examining the efficacy of the intervention. Data were collected at baseline (mean age $=12.5$ years, SD .45), and at +33 months. The 33 month follow up time was that determined by the study steering group for examination of the trial primary outcomes. Sample 1 consisted of 1,171 adolescents (40.82\% females, $2.56 \%$ unreported) attending secondary schools in Northern Ireland. Sample 2 consisted of 1,059 adolescents (52.79\% females, $1.32 \%$ unreported) attending secondary schools in Scotland. A form of parental opt-out consent was approved for the study, and participants also gave informed consent at each data collection point.

\section{Measures and demographics}

Twenty four items (see Appendix) from the Adolescent Stress Questionnaire [1] were administered (hereafter referred to the ASQ-Short). Abbreviations to the ASQ were developed by choosing the three items with the highest factor loadings in a Confirmatory Factor Analysis [6] across eight of the possible ten domains. The two domains not included in the study were stress of financial responsibility, and stress of emerging adult responsibility. These two factors were dropped based on both the relatively poor loadings of items, and the lack of internal consistency of factor scores reported by McKay et al. [6]. The second reason for dropping these factors was practical: The main research interests of the present cohort study were family and school-related issues. For each item, participants are asked, 'How stressful do you find' (e.g. living at home; going to school). Participants rated the level of stress experienced on a 5-point Likert-type scale ( $1=$ not stressful at all to $5=$ very stressful).

The Self-Efficacy Questionnaire for Children [13] contains 21 items assessing three domains of self-efficacy: (a) academic self-efficacy, (b) emotional self-efficacy, and (c) social self-efficacy. Each subscale consists of seven items, and respondents rate their competence in each self-efficacy domain on a 5-point Likert scale ( $1=$ not at all; $5=$ very well). Psychometric validity and internal consistency of SEQ-C subscale scores have elsewhere been reported $(\alpha>.80,[13] ; .77<\omega<.88,[25])$.

Information was gathered on gender, and on Free School Meals entitlement (FSM), an imperfect proxy for low-income families, and thus SES [14]. The proportions for FSM entitlement for Northern Ireland and Scotland were (respectively): Yes $=18.62 \% / 20.96 \% ;$ No $=70.62 \% / 58.55 \%$; Unsure $=$ $10.76 \% / 20.49 \%$.

\section{Statistical analyses}

All CFA models were developed using the Weighted Least Squares Means and Variances Adjusted (WLSMV) estimator in Mplus Version 7 [15], which is recommended for use with ordinal categorical data and is robust to non-normality [16]. There were a total of 137 missing data patterns when including both data collections. Because WLSMV is not robust to missing data, multiple imputation of missing data using Bayesian analysis was applied in Mplus as well $[17,18]$. In total, 10 imputed datasets were used. The following indices were used as fit guidelines: (a) Tucker-Lewis Index (TLI) and Comparative Fit Index (CFI) acceptable $\geq .90$ or excellent $\geq .95$, and (b) Root Mean Square Error of Approximation (RMSEA), acceptable $\leq .08$ or excellent $\leq .05$ [19]. Measurement invariance was scrutinized between the two data collections as well as between the genders, countries of residence, and socioeconomic statuses using multi-group CFA models: $\Delta C F I \leq .01$ [20]. To aid the interpretation of the regression analyses, we applied the guidelines of Ferguson [21]. Accordingly, for correlations, values $>.2$ were considered practically or meaningfully significant, with those $>.5$ considered moderate.

\section{Results}

None of the eight ASQ scale scores were skewed or kurtotic (Table 1). Broadly speaking, the internal consistency estimates were adequate, ranging from .67 to .86 . Only three internal consistency estimates fell below .70, all of which occurred at Time 1 , and in all three cases the values were $>.75$ by Time 2 . In fact, internal consistency estimates increased as students got older across all factors with the exception of scores associated with the stress of school attendance in Scotland.

Tables 2 and 3 show the eight factor structure fit the data well at both time points. With regard to ASQ measurement between data collections, differences in the fit of nested models suggested configural, metric, and scalar invariance. Furthermore, at both time points, configural, metric, and scalar invariance of scores were indicated by gender, country, and socioeconomic status. In other words, it appears as though the number of ASQ factors did not vary between groups (i.e., configural), the factor loadings did not differ substantially between groups (i.e., metric), and neither did the combination of the factor loadings and 
indictor/item intercepts (i.e., one group did not respond in a systematically higher way than the other; scalar). With regard to psychometrics at the item level, all factor loadings in both countries, and across both data collections, were all equal to or greater than 0.46 .

Correlations among ASQ and self-efficacy scores were consistent with theory (Table 4), and predictably negative. Of the 24 correlation coefficients observed at Time 1, 12 reached Ferguson's [21] practically significant threshold, with that number rising to 19 at Time 2 . It is further noted that stronger correlations were observed at follow-up than baseline, meaning that the relationship between self-efficacy and stress increases as students get older. Interestingly, at +33 months, emotional selfefficacy had the strongest relationship with each of the stress domains. Therefore, it seems as students get older their stress appears to be best explained by emotional self-efficacy regardless of the domain.

\section{General Discussion}

The robust nature of the psychometric findings herein suggests that adolescent stress resides in separate - though statistically related - domains of experience, eight of which are well accounted for by ASQ scores. ASQ scores also appear to capture the same eight stress constructs across key demographic groups, including gender, SES, and country of origin in the UK. That fact opens the door for researchers to compare stress between demographic groups, and to control for demographic covariates in studies on stress outcomes. Further still, the ASQ factor solution appears to be stable over time, which further suggests that the instrument is viable across a reasonable adolescent age range. Another interesting finding concerns the large internal consistency estimates for ASQ form factors. Elsewhere, Streiner [22] highlighted that Cronbach's $\alpha$ vales are directly influenced by the number of items used (such that it is more difficult to achieve a higher $\alpha$ with fewer items), and the fact that the majority of these values (and the omega estimates) were $>.70$ at both time points speaks to the reliability of ASQ scores.

Turning to convergent validity, correlations between ASQ scores and scores on self-efficacy support the domain specific nature of both constructs as well as the construct validity of ASQ scores. The age effect here is also interesting, expected and worthy of note. As the experience of stressors becomes consolidated with adolescents moving further into a developmental phase providing greater opportunities for the experience of stress, the chances of this experience impacting responses to assessments of health risk potential seems a plausible finding. However, self-efficacy - while a very central issue for adoles- cent health - is only a proxy measure of subsequent health risk.

The ASQ provides a domain specific and easy-to-administer scale to those wishing to examine stress more generally in adolescents, and offers an alternative to other existing measures of stress. However, the assessment of stress, is by its very nature complicated by the fact that stress resides not only in domains, but the experience of stress is also specifically felt in particular populations and in specific contexts. For example, the 20 -item revised Perceived Stress Questionnaire [23], which measures four dimensions of stress (worries, tension, joy, demands) may be particularly appropriate for use in clinical settings, while the Stress in General Scale was specifically developed to assess stress in the workplace. However, what is specific to the ASQ is the strong psychometric support for the assessment of stress at a particularly volatile time in life: adolescence [4]. It is noteworthy that, for the assessment of stress in some minority adolescent populations, longer scales are still being developed in order to assess the more nuanced dimensions of stress that are specific to those populations. For example, Scragher et al. [24] recently developed the 54-item, 10-factor Sexual Minority Adolescent Stress Inventory. Therefore, for those wishing to assess stress in more specific populations and contexts, the ASQ described herein may not be the inventory best suited to their needs.

The results of the present study need to be interpreted in the context of some limitations. Firstly, ASQ items were those that loaded best in a previous study [6], and while it may have made statistical sense to use these items, some of the excluded items might have theoretical value. Secondly, two of the factors from the ASQ were not used herein, again based on previous psychometric concerns, but also based on the fact that researchers were principally interested in family and school-related stressors. Finally, the properties of the short form were not tested against the longer ASQ. However, the rationale for using the shortened form was the danger of research fatigue in the context of the administration of a battery of instruments.

\section{Conclusion}

In conclusion, despite item choice limitations, the resultant 24-item scale is a viable instrument for the assessment of adolescent stress across eight domains, and provides a user-friendly assessment tool for researchers using multiple questionnaires in the same study. Results presented herein testify to the temporal stability, internal consistency, and psychometric validity of ASQ scores, and further, support previous studies evidencing the domain specific nature of stress in adolescence. 
Tables

Table 1: Descriptive Statistics for Multidimensional Adolescent Stress Questionnaire Scores.

\begin{tabular}{|c|c|c|c|c|c|c|c|c|}
\hline & \multicolumn{4}{|c|}{ Time 1} & \multicolumn{4}{|c|}{ Time 2} \\
\hline Home Life & 3.02 & 1.18 & .75 & .75 & 3.05 & 1.07 & .76 & .76 \\
\hline School Performance & 3.48 & 1.05 & .73 & .72 & 3.55 & 1.00 & .78 & .78 \\
\hline Romantic Relationships. & 1.97 & 1.03 & .73 & .75 & 2.14 & 1.03 & .75 & .78 \\
\hline Peer Pressure & 2.68 & 1.09 & .68 & .69 & 2.50 & 1.10 & .81 & .81 \\
\hline Teacher Interaction & 2.96 & 1.17 & .72 & .72 & 2.80 & 1.06 & .76 & .78 \\
\hline \multicolumn{9}{|l|}{ Scotland } \\
\hline Home Life & 2.97 & 1.19 & .73 & .72 & 3.30 & 1.01 & .77 & .78 \\
\hline School Performance & 3.27 & 1.06 & .68 & .67 & 3.63 & 0.99 & .76 & .76 \\
\hline School Attendance & 2.76 & 1.31 & .80 & .80 & 2.67 & 1.20 & .79 & .80 \\
\hline Romantic Relationships & 1.73 & .95 & .70 & .72 & 2.18 & 1.11 & .76 & .79 \\
\hline Peer Pressure & 2.54 & 1.08 & .67 & .69 & 2.45 & 1.13 & .78 & .78 \\
\hline Teacher Interaction & 2.93 & 1.17 & .73 & .74 & 3.06 & 1.11 & .77 & .76 \\
\hline
\end{tabular}

Note: $\mathrm{SD}=$ Standard Deviation; $\alpha=$ Alpha; $\omega^{h}=$ Omega Hierarchical

Table 2: Confirmatory Factory Analyses for ASQ-Short at Time 1.

\begin{tabular}{|c|c|c|c|c|c|c|}
\hline Model & $x 2 s-b$ & $d f$ & CFI & $\Delta \mathrm{CFI}$ & TLI & RMSEA \\
\hline CFA 8-Factor & $1788.943^{*}$ & 224 & 0.963 & -- & 0.955 & 0.056 \\
\hline \multicolumn{7}{|l|}{ Time } \\
\hline Configural & $4406.700 *$ & 496 & 0.953 & -- & 0.947 & 0.063 \\
\hline Metric & $4641.390 *$ & 512 & 0.950 & $0.003^{\mathrm{a}}$ & 0.946 & 0.063 \\
\hline Scalar & $4632.293^{*}$ & 536 & 0.950 & $0.003^{\mathrm{a}}$ & 0.949 & 0.062 \\
\hline \multicolumn{7}{|l|}{ Gender } \\
\hline Configural & $2012.601^{*}$ & 496 & 0.964 & -- & 0.960 & 0.053 \\
\hline Metric & 1979.049* & 512 & 0.966 & $0.002^{\mathrm{a}}$ & 0.963 & 0.051 \\
\hline Scalar & 1901.548* & 536 & 0.968 & $0.004^{a}$ & 0.967 & 0.048 \\
\hline \multicolumn{7}{|l|}{ Country } \\
\hline Configural & $2150.416^{*}$ & 496 & 0.961 & -- & 0.957 & 0.055 \\
\hline Metric & 2099.388* & 512 & 0.963 & $0.002^{\mathrm{a}}$ & 0.960 & 0.053 \\
\hline Scalar & $2110.563^{*}$ & 536 & 0.963 & $0.002^{a}$ & 0.962 & 0.051 \\
\hline \multicolumn{7}{|c|}{ Socioecon. Status } \\
\hline Configural & $1554.674^{*}$ & 796 & 0.970 & -- & 0.967 & 0.048 \\
\hline Metric & $1512.859^{*}$ & 512 & 0.972 & $0.002^{\mathrm{a}}$ & 0.969 & 0.046 \\
\hline Scalar & $1358.988^{*}$ & 536 & 0.977 & $0.007^{a}$ & 0.976 & 0.040 \\
\hline
\end{tabular}

Note: ${ }^{*} p \leq .01 . \mathrm{s}-\mathrm{b}$ : Santorra-Bentler; DF: Degrees of freedom; CFI: Comparative fit index; TLI: Tucker lewis index; RMSEA: Root mean Squared error of approximation; A: Cheung and Rensvold's (2002); $\triangle \mathrm{CFI} \leq .01$ has been met, indicating measurement invariance. 
Table 3: Confirmatory Factory Analyses for ASQ-Short at Time 2.

\begin{tabular}{|l|l|l|l|l|l|l|}
\hline Model & X2s-b & Df & CFI & $\Delta$ CFI & TLI & RMSEA \\
\hline CFA 8-Factor & $2391.937^{*}$ & 224 & 0.957 & -- & 0.948 & 0.066 \\
\hline Gender & & & & & \\
\hline Configural & 4369.128 & 496 & 0.919 & -- & 0.909 & 0.085 \\
\hline Metric & 4352.299 & 512 & 0.919 & $0.000^{\mathrm{a}}$ & 0.913 & 0.083 \\
\hline Scalar & 4132.431 & 536 & 0.924 & $0.005^{\mathrm{a}}$ & 0.922 & 0.078 \\
\hline Country & & & & & 0.056 \\
\hline Configural & 2231.954 & 496 & 0.966 & -- & 0.962 & 0.055 \\
\hline Metric & 2224.936 & 512 & 0.967 & $0.001^{\mathrm{a}}$ & 0.964 & 0.050 \\
\hline Scalar & 2030.272 & 536 & 0.971 & $0.005^{\mathrm{a}}$ & 0.970 & \\
\hline Socioecon. Status & & & & & & 0.051 \\
\hline Configural & 1690.419 & 496 & 0.971 & -- & 0.968 & 0.049 \\
\hline Metric & 1688.081 & 512 & 0.972 & $0.001^{\mathrm{a}}$ & 0.969 & 0.045 \\
\hline Scalar & 1545.649 & 536 & 0.976 & $0.005^{\mathrm{a}}$ & 0.975 & \\
\hline
\end{tabular}

Note:* $p \leq .01$. s-b: Santorra-bentler; DF: degrees of freedom; CFI: Comparative fit index; TLI: Tucker lewis index; RMSEA : Root mean squared error of approximation; $A=$ Cheung and Rensvold's (2002); $\triangle C F I \leq .01$ has been met, indicating measurement invariance.

Table 4: Correlations Among Multidimensional Adolescent Stress Questionnaire and Self-Efficacy Scores at both times.

\begin{tabular}{|l|c|c|c|c|c|c|}
\hline & \multicolumn{4}{|c|}{ Time 1 } & \multicolumn{3}{c|}{ Time 2 } \\
\hline & $\begin{array}{c}\text { Social Self } \\
\text { Efficacy }\end{array}$ & $\begin{array}{c}\text { Emotional } \\
\text { Self Efficacy }\end{array}$ & $\begin{array}{c}\text { Academic } \\
\text { Self Efficacy }\end{array}$ & $\begin{array}{c}\text { Social Self } \\
\text { Efficacy }\end{array}$ & $\begin{array}{c}\text { Emotional } \\
\text { Self Efficacy }\end{array}$ & $\begin{array}{c}\text { Academic } \\
\text { Self Efficacy }\end{array}$ \\
\hline Home Life & $-0.10^{*}$ & $-0.29^{*}$ & $-0.19^{*}$ & $-0.23^{*}$ & $-0.46^{*}$ & $-0.22^{*}$ \\
\hline School Performance & $-0.10^{*}$ & $-0.29 *$ & $-0.30^{*}$ & $-0.20^{*}$ & $-0.44^{*}$ & $-0.28^{*}$ \\
\hline School Attendance & $-0.13^{*}$ & $-0.19^{*}$ & $-0.42^{*}$ & $-0.20^{*}$ & $-0.30^{*}$ & $-0.45^{*}$ \\
\hline Romantic Relationships. & -0.07 & $-0.17^{*}$ & $-0.26^{*}$ & $-0.18^{*}$ & $-0.30^{*}$ & $-0.26^{*}$ \\
\hline Peer Pressure & $-0.24 *$ & $-0.31^{*}$ & $-0.12^{*}$ & $-0.40^{*}$ & $-0.45^{*}$ & $-0.11^{*}$ \\
\hline Teacher Interaction & -0.08 & $-0.21^{*}$ & $-0.34^{*}$ & $-0.14^{*}$ & $-0.36^{*}$ & $-0.34^{*}$ \\
\hline Future Uncertainty & $-0.13^{*}$ & $-0.29^{*}$ & $-0.13^{*}$ & $-0.22^{*}$ & $-0.49 *$ & $-0.11^{*}$ \\
\hline School Leisure & -0.06 & $-0.21^{*}$ & $-0.22^{*}$ & $-0.14^{*}$ & $-0.29 *$ & $-0.23^{*}$ \\
\hline
\end{tabular}

\section{References}

1. Byrne DG, Davenport SC, Mazanov J. Profiles of adolescent stress: The development of the adolescent stress questionnaire (ASQ). Journal of Adolescence. 2007; 30: 393-416.

2. Moksnes UK, Espnes GA, Haugan G. Stress, sense of coherence and emotional symptoms in adolescents. Psychology \& Health. 2014; 29: 32-49.

3. Darviri C, Legaki PE, Chatzioannidou P, Gnardellis C, Kraniotou C, Tigani $X$, et al. Adolescent Stress Questionnaire: Reliability and validity of the Greek version and its description in a sample of high school (lyceum) students. Journal of Adolescence. 2014; 37: 1373-1377.

4. Moksnes UK, Espnes GA. Evaluation of the Norwegian version of the AdolescentStressQuestionnaire (ASQ-N): Factorial validity across samples. Scandinavian Journal of Psychology. 2011; 52: 601-608.

5. De Vriendt T, Clays E, Moreno LA, Bergman P, Vicente-Rodriguez G, Nagy E, et al. HELENA Study Group. Reliability and validity of the Adolescent Stress Questionnaire in a sample of European adolescents - the HELENA study. BMC Public Health. 2011; 11: 717.

6. McKay MT, Percy A, Byrne DG. Support for the Multidimensional Adolescent Stress Questionnaire in a sample of adolescents in the United Kingdom. Stress and Health. 2016; 32: 12-19.

7. Moksnes UK, Bradley Eilertsen ME, Lazarewicz M. The association between stress, self esteem, and depressive symptoms in adolescents. Scandinavian Journal of Psychology. 2016; 57: 22-29.

8. Moksnes UK, Løhre A, Lillefjell M, Byrne DG, Haugan G. The associations between school stress, life satisfaction, and depressive symptoms in adolescents: life satisfaction as a potential mediator. Social Indicators Research. 2016; 125: 339-357.

9. McKay MT, Cole JC. Adolescent drinking and adolescent stress: A domain-specific relationship in Northern Irish school children. Journal of Youth Studies. 2012; 16: 237-256.

10. McKay MT, Dempster M. Byrne DG. An examination of the relationship between self-efficacy and stress in adolescents: The role of gender and self-esteem. Journal of Youth Studies. 2014; 
17: 1131-1151.

11. Newby-Fraser E, Schlebusch L. Social support, self-efficacy and assertiveness as mediators of student stress. Psychology: A Journal of Human Behavior. 1997; 34: 61-69.

12. Shelley M, Pakenham K. External Health Locus of Control and General Self- Efficacy: Moderators of Emotional Distress among University Students. Australian Journal of Psychology. 2004; 56: 191-199.

13. Muris P. A brief questionnaire for measuring self-efficacy in youths. Journal of Psychopathology and Behavioral Assessment. 2001; 23: 145-149.

14. Hobbs $\mathrm{G}$, Vignoles A. Is free school meal status a valid proxy for socio-economic status (in schools research)? London: Centre for the Economics of Education. 2007.

15. Muthén BO, Muthén LK. Mplus user's guide (7th edition.). Los Angeles, CA. $1998-2012$.

16. Wang J, Wang X. Structural equation modeling: Applications in Mplus. West Sussex: United Kingdom: John Wiley \& Sons. 2012.

17. Rubin DB. Inference and missing data. Biometrika. 1976; 63: 581-592.

18. Schafer JL. Analysis of incomplete multivariate data. London: Chapman \& Hall. 1997.

19. Marsh HW, Hau K, Wen Z. In search of golden rules: Comment on hypothesis-testing approaches to setting cutoff values for fit indexes and dangers in overgeneralizing Hu and Bentler's (1999) findings. Structural Equation Modeling: A Multidisciplinary Journal. 2004; 11: 37-41.

20. Cheung GW, Rensvold RB. Evaluating goodness-of- fit indexes for testing measurement invariance. Structural Equation Modeling: A Multidisciplinary Journal. 2002; 9: 233-255.
21. Ferguson $\mathrm{CJ}$. An effect size primer: $\mathrm{A}$ guide for clinicians and researchers. Professional Psychology: Research and Practice. 2009; 40: 532-538.

22. Streiner DL. Starting at the beginning: An introduction to coefficient alpha and internal consistency. Jou rnal of Personality Assessment. 2003; 80: 99-103.

23. Fliege $H$, Rose $M$, Arck $P$, Walter $O$, Kocalevent RD, Weber $C$, et al. The Perceived Stress Questionnaire (PSQ) Reconsidered: Validation and Reference Values From Different Clinical and Healthy Adult Samples. Psychosomatic Medicine. 2005; 67: 78-88.

24. Schrager SM, Goldbach JT, Mamey MR. Development of the Sexual Minority Adolescent Stress Inventory. Frontiers in Psychology. 2018; 9: 319.

25. Wells KE, McKay MT, Morgan GB, Worrell FC. Time attitudes predict changes in adolescent self-efficacy: A 24-month latent transition mover-stayer analysis. J Adolescence. 2018; 62: 27-37.

26. McKay M, Agus A, Cole J, Doherty P, Foxcroft D, Harvey S, Murphy L, Percy A, Sumnall H. Steps Towards Alcohol Misuse Prevention Programme (STAMPP): A school-based and community-based cluster randomised controlled trial. BMJ Open. 2018; 8(3): e019722. 